\title{
Modelo de gestión de carteras de Markowitz usando algoritmos genéticos
}

Markowitz portfolio management model using genetic algorithms

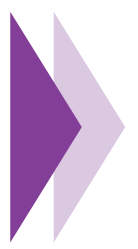

\section{Santiago Arévalo González}

Ingeniero Electrónico. Maestrando en Ingeniería Biomédica. Perteneciente al grupo de investigación en Cibernética de la Universidad Nacional de Entre Ríos (Argentina)

E-mail: sarevalog@correo.udistrital.edu.co

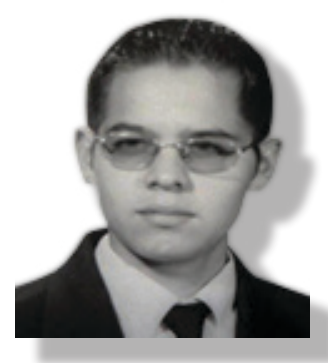




\section{RESUMEN}

Con su artículo de 1952, Harry Markowitz simplificó notablemente el problema de selección de inversiones en el mercado accionario. En este artículo se introduce al lector en la propuesta de Markowitz y se propone un algoritmo genético para deducir la combinación de dos activos (cartera), que obtenga la más alta rentabilidad para su inversión y al mismo tiempo obtener el menor riesgo. En la última parte se aplica el algoritmo genético en dos casos de estudio reales con registros mensuales obtenidos entre 2009 y 2013.

Palabras Claves: Algoritmo genético simple, Teoría de carteras, modelo de Markowitz, Teoría moderna de carteras, economía financiera.

\section{ABSTRACT}

In 1952, with his article, Harry Markowitz, he greatly simplified the problem to choice the correct investing in a stock market. In this work, the reader is introduced in the Markowitz's proposal and it is proposed a genetic algorithm for computing a portfolio that reduce risk and maximize return between two assets. In the last part, the genetic algorithm is applied in two real case studies with monthly records obtained between 2009 and 2013.

Keywords: Simple genetic algorithm, Portfolio theory, Markowitz's theory, Modern portfolio theory (MPT), financial economy.

\section{Introducción}

El mercado financiero resulta muy atractivo para inversionistas y corredores de bolsa, pues permite tener altos rendimientos de dinero en poco tiempo comparado con otro tipo de negocios. Sin embargo el riesgo tomado es alto, muchas veces los sentimientos del experto afectan la forma de inversión, haciendo que la predicción que se tenía no sea la correcta (Roldán Hernández y Lugo Currea, 2010).

Anterior al trabajo de Markowitz, los inversores solamente prestaban atención en maximizar el nivel esperado de retornos, es decir que un inversor calcularía simplemente el grado esperado de rendimientos de un conjunto de activos y luego invertiría todo su dinero en aquel activo que proporcione la mayor rentabilidad esperada.

En su teoría moderna de selección de cartera, Markowitz (1952) demostró que los inversores deberían actuar de un modo totalmente diferente. Estos deben optar por portafolios de varios activos en vez que invertir en un solo activo. Dicho coloquialmente, "no poner todos los huevos en la misma canasta".

Siguiendo este consejo de mantener un portafolio de activos (diversificación), un inversor puede reducir el nivel de riesgo al cual está exponiéndose, mientras que mantiene el nivel esperado de rentabilidad. En términos financieros, la diversificación se puede definir como una estrategia de inversión que puede reducir el riesgo del mercado al combinar una variedad de inversiones, como acciones y bonos, los cuales probablemente no se muevan en la misma dirección al mismo tiempo (Gordon, 2003), y el riesgo se define como el grado de incertidumbre sobre la tasa de rendimiento y/o valor del principal de una inversión. Como un portafolio o cartera no es nada más que un conjunto de activos, el problema se "reduce" a seleccionar la mejor cartera entre un conjunto de posibles carteras. Un inversor racional siempre pretenderá obtener la más alta rentabilidad para su inversión, y al mismo tiempo esa rentabilidad asuma el menor riesgo posible (menor desviación típica) (Gordon, 2003). El resultado más importante del enfoque de Markowitz es que permite deducir combinaciones de activos (portafolios) que simultáneamente cumplen con dos condiciones:

- Tienen la varianza mínima dentro de todas las combinaciones posibles que tienen un rendimiento esperado dado.

- Tienen el rendimiento esperado máximo dentro de todas las combinaciones posibles que tienen una varianza dada.

\section{Modelo de gestión Markowitz}

El modelo se plantea para la combinación de dos supuestos activos. Se establece el objetivo de 
fijar el menú de las posibles combinaciones de rentabilidad $R$, siendo el peso asignado al activo 1 R1 la variable sobre la cual se tiene capacidad de decisión.

$$
R=\omega \square R 1+(1-\omega) \square R 1
$$

El riesgo que se puede expresar como la varianza de la cartera de la siguiente manera:

(2) Riesgo $=\operatorname{var}(R)$

(3)

$$
\text { Riesgo }=\left(\omega^{2} \square \sigma_{1}^{2}+(1-\omega)^{2} \square \omega_{2}^{2}+2 \square \omega \square(1-\omega) \square \omega_{1} \square \omega_{2} \square \omega_{12}\right)^{1 / 2)}
$$

En este modelo: $\omega$ es el porcentaje de inversión, $\sigma 1, \sigma 2$ y $\sigma 12$ son la desviación típica del activo 1 , 2 y la covarianza de 1 en 2 respectivamente. Al ir cambiando el valor de $\omega$, se obtienen pares de rentabilidad y riesgo, que representan las distintas alternativas posibles, comprendiendo de esta manera al conjunto factible. Se resalta que la solución de Markowitz pretende reducir el riesgo, en otras palabras un máximo nivel de rentabilidad que se puede alcanzar con el menor riesgo.

\section{Algoritmo genético}

Se propone un algoritmo genético clásico que se puede esquematizar como sigue:

\section{Iniciar(Población) \\ MejorFitness=Evaluar (Población) \\ Mientras(Generación=MaxGeneración) \\ Selección=Seleccionar (Población) \\ Población=Cruce y Mutación(Población)}

Fin mientras

Para la implementación del algoritmo ha sido desarrollada y evaluada con el software matemático MATLAB de Mathworks, versión R2013b.

\subsection{Representación de los individuos}

El porcentaje de capital invertido en la acción 1 es un número que variara de o a 1 (espacio fenotípico).
Se opta emplear cadenas binarias y el espacio de búsqueda sea dado por una representación binaria de 7 bits.

Se aplica un factor de escala y truncamiento para convertir al real $\mathrm{x}$ en un entero $\mathrm{X}$ perteneciente al rango $\left[0,2^{\wedge}(n-1)\right]$ y se convierte el entero $X$ en el número binario (D. Milone, H. L. Rufiner y R. Acevedo, 2009).

\subsection{Criterio de evaluación y aptitud}

Para encontrar el porcentaje de capital $\left(\omega^{*}\right)$ óptimo a invertir en cada activo, se deriva la función de riesgo (Ecuación (3)) con respecto a $\omega$ y se iguala a cero

(4)

$\frac{\partial(\operatorname{Var}(R))}{\partial \omega}=E \operatorname{vat}(\omega)=\omega * \sigma_{1}^{2}+\omega * \operatorname{sigma} a_{2}^{2}-2 * \omega * \sigma_{12}-\sigma_{1}^{2}+\sigma_{12}$

Los individuos que acerquen la ecuación (4) a cero serán los mejores individuos como potenciales soluciones al problema. Por ende, los individuos de cada generación que tengan el menor valor serán recompensados con una mayor aptitud. Esta opción proporciona una referencia visible común para todas las aptitudes, pero exige que la función sea estrictamente positiva, situación que se expone en la sección 3.4 desplazamiento.

\subsection{Parametrización del algoritmo}

Se ha realizado una gran cantidad de pruebas, sobre una amplia variedad de problemas de búsqueda y optimización, con la intención de determinar rangos de buen funcionamiento para los parámetros del Algoritmo Genético Simple (Davis, 1991; Michalewicz, 1994; Schaffer, 1989). Aunque los resultados son dependientes en mayor o menor medida del problema en particular se escogieron los parámetros siguiendo los siguientes criterios:

Tamaño de la población: en términos generales, la población inicial debe ser lo más variada posible. Es necesario que la distribución de aptitudes sea uniforme para evitar la convergencia prematura, y también es necesario que haya variedad de esquemas. El tamaño de la población varía 
habitualmente entre 50 y 100 individuos; valores menores suelen plantear graves problemas de convergencia prematura y valores mayores requieren un gran esfuerzo computacional sin obtener mejoras apreciables (Pérez Serrada, 1996). Se evaluaron diferentes poblaciones iniciales, la evaluación de desempeño en los diferentes ejemplos muestra que los mejores resultados se observan cuando la población inicial varía entre 60 y 150 individuos. En el algoritmo final se eligió una población inicial de 100 elementos.

Probabilidad de mutación: las mejores prestaciones se obtienen en general, con tasas de cruce que varían entre el $20 \%$ y el $60 \%$ y tasas de mutación entre el $0.1 \%$ y el $5 \%$. Aunque no existe un criterio particular para escoger un valor determinado, se eligió una probabilidad de mutación del $5 \%$ y una tasa de cruce del 20\% (número de progenitores a seleccionar), ya que diferentes autores han recomendado estos parámetros (Grefenstette, 1995; Levine, 1995; Smith et al, 1995; Hunter, 1995).

Generaciones máximas: el máximo número de iteraciones depende de la precisión especificada y varía mucho de un problema a otro; es común usar una iteración máxima de 50 para problemas de evaluación compleja y una iteración máxima de 1.000 para problemas de evaluación sencilla. Para el presente planteamiento se decidió usar una iteración máxima de 200.

\subsection{Desplazamiento}

Se debe aceptar que pueden resultar ponderadores negativos en (4), lo que implica reconocer la posibilidad de que un portafolio puede estar constituido por algunos componentes que en realidad son pasivos, algo que se debe, no que se posee. Esto es perfectamente factible en aquellos casos en que el carácter de las instituciones financieras permite las ventas en corto, es decir, la venta de un activo que ha sido tomado en préstamo y la utilización de este ingreso para la compra de otro activo. En este caso, el portafolio de inversión estará formado por algunos componentes cuyas ponderaciones son negativas y otros con ponderaciones superiores a la unidad (Lasa, 2009). Con el propósito de hacer que la función de aptitud devuelva únicamente valores positivos se realiza un desplazamiento de la función de la siguiente manera.

(5) Aptitud (individuo) $=1 /$ fitness (individuo)

(6) $\operatorname{Aptitud}(\omega)=1+\operatorname{Eval}(v)$

\section{Casos de estudio}

\subsection{Samsung- Apple}

Se analizaron los dos activos de la bolsa de valores estadounidense: Apple Inc. y Samsung Electronics Co. Ltd. Ya que es un portafolio donde sus componentes tienen alto nivel de relación. Para el análisis se tomó el intervalo de noviembre de 2009 a junio de 2013 con un total de 44 precios de cierres históricos que se pueden encontrar en Yahoo Finance (2013). Se obtienen los diferentes coeficientes de media, varianza, los coeficientes de covarianza y correlación entre los activos. Estos coeficientes son obtenidos mediante el cómputo de los rendimientos logarítmicos y están representados en la Tabla 1.

Tabla 1: Coeficientes de variables del portafolio AppleSamsung.

\begin{tabular}{|l|l|l|l|}
\hline \multicolumn{2}{|c|}{ APPLE } & \multicolumn{2}{c|}{ SAMSUNG } \\
\hline $\begin{array}{l}\text { Rentabilidad } \\
\text { mensual } \\
\text { promedio }\end{array}$ & $1.97 \%$ & $\begin{array}{l}\text { Rentabilidad } \\
\text { mensual } \\
\text { promedio }\end{array}$ & $1.89 \%$ \\
\hline $\begin{array}{l}\text { Desviación } \\
\text { estándar }\end{array}$ & 0.075047602 & $\begin{array}{l}\text { Desviación } \\
\text { estándar }\end{array}$ & 0.072073224 \\
\hline Varianza & 0.005632143 & Varianza & 0.005632143 \\
\hline Covarianza & & 0.001755345 \\
\hline Correlación & 0.780305594 \\
\hline
\end{tabular}

Se valida el algoritmo en el software Matlab de Matworks. Se ingresan los datos en el algoritmo y en el transcurso de 200 generaciones se obtiene que la mejor forma de invertir es 0.4961, esto significa que se recomienda invertir en Apple el $49.61 \%$ del dinero y el resto en Samsung. Esta 
respuesta es comparada con el resultado obtenido de la forma clásica donde se evalúan cada una de las posibilidades de cartera para obtener la frontera de portafolios; correspondiente a la cartera 11 en la Tabla 2.

Tabla 2: Fronteras eficientes portafolio Apple-Samsung.

\begin{tabular}{|l|c|c|c|c|}
\hline & $\mathrm{W}$ & $1-\mathrm{W}$ & Riesgo & Rentabilidad \\
\hline Cartera1 & $0 \%$ & $100 \%$ & $0.563 \%$ & $1.89 \%$ \\
\hline Cartera2 & $5 \%$ & $95 \%$ & $0.510 \%$ & $1.90 \%$ \\
\hline Cartera3 & $10 \%$ & $90 \%$ & $0.463 \%$ & $1.90 \%$ \\
\hline Cartera4 & $15 \%$ & $85 \%$ & $0.422 \%$ & $1.91 \%$ \\
\hline Cartera5 & $20 \%$ & $80 \%$ & $0.385 \%$ & $1.91 \%$ \\
\hline Cartera6 & $25 \%$ & $75 \%$ & $0.355 \%$ & $1.91 \%$ \\
\hline Cartera7 & $30 \%$ & $70 \%$ & $0.330 \%$ & $1.92 \%$ \\
\hline Cartera8 & $35 \%$ & $65 \%$ & $0.311 \%$ & $1.92 \%$ \\
\hline Cartera9 & $40 \%$ & $60 \%$ & $0.297 \%$ & $1.93 \%$ \\
\hline Cartera10 & $45 \%$ & $55 \%$ & $0.288 \%$ & $1.93 \%$ \\
\hline Cartera11 & $50 \%$ & $50 \%$ & $0.286 \%$ & $1.93 \%$ \\
\hline Cartera12 & $55 \%$ & $45 \%$ & $0.288 \%$ & $1.94 \%$ \\
\hline Cartera13 & $60 \%$ & $40 \%$ & $0.297 \%$ & $1.94 \%$ \\
\hline Cartera14 & $65 \%$ & $35 \%$ & $0.311 \%$ & $1.94 \%$ \\
\hline Cartera15 & $70 \%$ & $30 \%$ & $0.330 \%$ & $1.95 \%$ \\
\hline Cartera16 & $75 \%$ & $25 \%$ & $0.355 \%$ & $1.95 \%$ \\
\hline Cartera17 & $80 \%$ & $20 \%$ & $0.385 \%$ & $1.96 \%$ \\
\hline Cartera18 & $85 \%$ & $15 \%$ & $0.422 \%$ & $1.96 \%$ \\
\hline Cartera19 & $90 \%$ & $10 \%$ & $0.463 \%$ & $1.96 \%$ \\
\hline Cartera20 & $95 \%$ & $5 \%$ & $0.510 \%$ & $1.97 \%$ \\
\hline Cartera21 & $100 \%$ & $0 \%$ & $0.563 \%$ & $1.97 \%$ \\
\hline
\end{tabular}

\subsection{Ecopetrol-Isagen}

Se planteó la inversión de un portafolio constituido por dos acciones colombianas: Ecopetrol e Isagen, los históricos se obtuvieron de la Bolsa de Valores de Colombia (2013). Para el análisis se tomó el intervalo de enero de 2013 a mayo de 2013 con un total de 102 precios de cierres históricos diarios (días hábiles). La Tabla 3 expone el resumen de las variables resultantes.
Tabla 3: Coeficientes de variables portafolio ISAGENECOPETROL.

\begin{tabular}{|l|l|l|l|}
\hline \multicolumn{2}{|c|}{ ISAGEN } & \multicolumn{2}{c|}{ ECOPETROL } \\
\hline $\begin{array}{l}\text { Rentabilidad } \\
\text { mensual } \\
\text { promedio }\end{array}$ & $-0.058 \%$ & $\begin{array}{l}\text { Rentabilidad } \\
\text { mensual } \\
\text { promedio }\end{array}$ & $0.287 \%$ \\
\hline $\begin{array}{l}\text { Desviación } \\
\text { estándar }\end{array}$ & 0.000147715 & $\begin{array}{l}\text { Desviación } \\
\text { estándar }\end{array}$ & 0.000199734 \\
\hline Varianza & 0.0121538 & Varianza & 0.014132738 \\
\hline Covarianza & 0.072008733 \\
\hline Correlación & $1.22462 E-05$ \\
\hline
\end{tabular}

Se ingresan los datos en el algoritmo y en el transcurso de 200 generaciones se obtiene que la mejor forma de invertir es 0.4252 , es decir que si fuese posible invertir en esas dos empresas, la mejor manera seria invertir el $42.52 \%$ del dinero en ISAGEN y el resto en ECOPETROL, se comparan con los portafolios eficientes como se observa en la Tabla 4, Cartera 9.

Tabla 4: Fronteras eficientes portafolio ISAGENECOPETROL.

\begin{tabular}{|l|c|c|c|c|}
\hline & W & $1-\mathrm{W}$ & Riesgo & Rentabilidad \\
\hline Cartera1 & $0 \%$ & $100 \%$ & $0.01477 \%$ & $-0.06 \%$ \\
\hline Cartera2 & $5 \%$ & $95 \%$ & $0.01407 \%$ & $-0.04 \%$ \\
\hline Cartera3 & $10 \%$ & $90 \%$ & $0.01346 \%$ & $-0.02 \%$ \\
\hline Cartera4 & $15 \%$ & $85 \%$ & $0.01296 \%$ & $-0.01 \%$ \\
\hline Cartera5 & $20 \%$ & $80 \%$ & $0.01256 \%$ & $0.01 \%$ \\
\hline Cartera6 & $25 \%$ & $75 \%$ & $0.01226 \%$ & $0.03 \%$ \\
\hline Cartera7 & $30 \%$ & $70 \%$ & $0.01206 \%$ & $0.05 \%$ \\
\hline Cartera8 & $35 \%$ & $65 \%$ & $0.01196 \%$ & $0.06 \%$ \\
\hline Cartera9 & $40 \%$ & $60 \%$ & $0.01197 \%$ & $0.08 \%$ \\
\hline Cartera10 & $45 \%$ & $55 \%$ & $0.01208 \%$ & $0.10 \%$ \\
\hline Cartera11 & $50 \%$ & $50 \%$ & $0.01229 \%$ & $0.11 \%$ \\
\hline Cartera12 & $55 \%$ & $45 \%$ & $0.01260 \%$ & $0.13 \%$ \\
\hline Cartera13 & $60 \%$ & $40 \%$ & $0.01301 \%$ & $0.15 \%$ \\
\hline Cartera14 & $65 \%$ & $35 \%$ & $0.01352 \%$ & $0.17 \%$ \\
\hline Cartera15 & $70 \%$ & $30 \%$ & $0.01414 \%$ & $0.18 \%$ \\
\hline
\end{tabular}




\begin{tabular}{|l|c|c|c|c|}
\hline Cartera16 & $75 \%$ & $25 \%$ & $0.01486 \%$ & $0.20 \%$ \\
\hline Cartera17 & $80 \%$ & $20 \%$ & $0.01568 \%$ & $0.22 \%$ \\
\hline Cartera18 & $85 \%$ & $15 \%$ & $0.01660 \%$ & $0.23 \%$ \\
\hline Cartera19 & $90 \%$ & $10 \%$ & $0.01762 \%$ & $0.25 \%$ \\
\hline Cartera20 & $95 \%$ & $5 \%$ & $0.01875 \%$ & $0.27 \%$ \\
\hline Cartera21 & $100 \%$ & $0 \%$ & $0.01997 \%$ & $0.29 \%$ \\
\hline
\end{tabular}

\section{Error}

Se determina el error entre la respuesta del algoritmo genético y la solución por la curva de fronteras eficientes a través del error cuadrático medio en los dos casos evaluados.

(7)

$$
E_{p 1}[(T-\theta)]=(0.5-0.4961)^{2}=0,00001521=0.001521
$$

(8)

$$
E_{p 2}[(T-\theta)\}=(0.4-0.4252)^{\hat{2}}=0.0635
$$

\section{Conclusiones}

Los algoritmos genéticos simples se adaptan fácilmente a problemas que usan modelos determinísticos, modificando ligeramente el algoritmo este convergerá exitosamente en la solución.

Diferentes variables modifican la cartera que genera una máxima rentabilidad; cambios en las políticas monetarias, catástrofes climatológicas, guerras internas y externas, por mencionar algunas, afectan de manera contundente su gestión. Es por ello que en este estudio se buscó una manera para analizar el modelo de gestión de cartera de Markowitz de una manera que se pudiese adaptar en el tiempo a medida que cambiaran estos parámetros.

Se pudo observar a través de los dos casos de estudio que la aproximación entregada por el algoritmo genético, se acercó con un error insignificante al resultado obtenido por la función de fronteras eficientes, lo que valida al mismo. Si bien el modelo se evaluó a través de una cartera de dos elementos, éste se puede adaptar a una cartera de múltiples activos y ser usado en tiempo real en la bolsa de acciones usando registros históricos por hora o minuto en vez de mensuales.

\section{REFERENCIAS}

Bedoya Villegas, J. A. (2011). Aplicación de la teoría Markowitz. Medellín: Universidad Nacional de Colombia.

Bolsa de Valores de Colombia. (Último acceso: junio 2013) En línea. Recuperado de: http:// www.bvc.com.co.

Davis, L. (1991). Handbook of Genetic Algorithms. New York: Van Nostrand Reinhold.

Gordon, A. (2003). Fundamentals of Investments/ Fundamentos de Inversiones. New York: Pearson.

Grefenstette, J. J. (1990). A User's Guide to GENESIS.

Hunter, A. (1995). Sugal Programming Manual.

Lasa, A. J. (2009). Construcción de una frontera eficiente de activos financieros en México. Denarius. 131-153 .

Levine, D. (1995). User's Guide to the PGA Pack Parallel Genetic Algorithm Library.

Markowitz, H. M. (1959). The Theory and Practice of Investment Management: Asset Allocation, Valuation, Portfolio Construction, and Strategies. New Jersey: John Wiley \& Sons.

Michalewicz, Z. (1994). Genetic Algorithms + Data Structures = Evolution Programs. Charlotte: Springer-Verlag..

Milone, D., Rufiner, H. L. y Acevedo, R. (2009). Introducción a las señales y los sistemas discretos. Concepción del Uruguay, Entre Ríos: EDUNER. 
Pérez Serrada, A. (1996). Una introducción a la computación evolutiva.

Roldán Hernández, A. F. y Lugo Currea, N. A.. (2010). Predicción del Valor de la Acción de ETB en la Bolsa de Acciones de Colombia mediante sistemas difusos. Bogotá: Universidad Distrital Francisco José de Caldas.

Shaffer, J. D. (ed.) (1989). Proceedings of the Thrid International Conference on Genetic Algorithms. New York: Morgan Kaufmann Publishers.

Smith, R. E., et al. (1995). SGA-C: A C-Language Implementation of a Simple Genetic Algorithm. The Clearinghouse for Genetic Algoritms, Tuscaloosa: The University of Alabama.

Yahoo Finance. (Último acceso: junio 2013) Recuperado de: http://finance.yahoo.com/. 